\title{
Do It Yourself solution of Internet of Things Healthcare System: Measuring body parameters and environmental parameters affecting health
}

\author{
Mirjana Maksimović ${ }^{1 *}$, Vladimir Vujović ${ }^{1} \&$ Branko Perišić $^{2}$ \\ ${ }^{1}$ University of East Sarajevo, BOSNIA AND HERZEGOVINA \\ ${ }^{2}$ University of Novi Sad, SERBIA
}

\begin{abstract}
The rapid advancements in information and communications technologies (ICT) and the increasing number of smart things shift an old-fashioned healthcare system to a model better suited for a population of the $21 \mathrm{st}$ century. New healthcare approaches based on Internet of Things (Io'T)/Internet of Medical Things (IoMT) powered systems make health monitoring, diagnostics and treatment more personalized, timely and convenient, enabling a global approach to the healthcare system infrastructure development. Commercial systems in this area exist in various forms but usually do not fit the general patient needs, and those that do are usually economically unacceptable due to the high operational and development costs. Do It Yourself (DIY) healthcare, including mobile applications and consumer medical devices, nowadays is the top healthcare trend. Therefore, this paper, based on well-known low-cost technologies, presents a DIY IoMT solution for observing human vital parameter as well as environmental factors affecting health.
\end{abstract}

\begin{abstract}
Keywords
Do It Yourself

healthcare, Internet of Things, Internet of Medical Things, self-monitoring devices,

Raspberry Pi

Received: 8 Jan 2016

Revised: 27 Jan 2016

Accepted: 20 Feb 2016
\end{abstract}

DOI: $10.20897 /$ lectito. 201607

\section{INTRODUCTION}

Health is the fundamental capability humans require to perceive, feel, and act effectively, and as such, it represents a primary element in the development of the individual, but also of the environment humans belongs to (Maksimović et al., 2015). The role and the importance of healthcare systems in the quality of life and social welfare in modern society have been broadly well recognized. To improve human health and wellbeing is the ultimate goal of any economic, technological and social development. Therefore, population's health has become a state responsibility, affecting also the competitiveness of modern economies. In the years preceding the economic crisis, health spending outpaced the rest of the economy. But in the recent years, it is evident that with increases in health spending in line with overall economic development, health expenditure as a percentage of GDP (Gross Domestic Product) has remained stable. Many European countries saw further reductions in health spending in 2013, according to OECD Health Statistics 2015 (Figure 1). Most countries in the European Union reported real per capita health spending below the levels of 2009. Outside of Europe, health spending has been rising at about $2.5 \%$ per year since 2010 (OECD Health Statistics, 2015). Growth in average annual healthcare spending 2014 - 2018 is expected to range from $2.4 \%$ in Western Europe to $4.9 \%$ in North America; and from 8.1\% in Asia and Australia to $8.7 \%$ in the Middle East and Africa (Deloitte, n.d.). Top Healthcare development areas for 2015 are presented in Figure 2. 


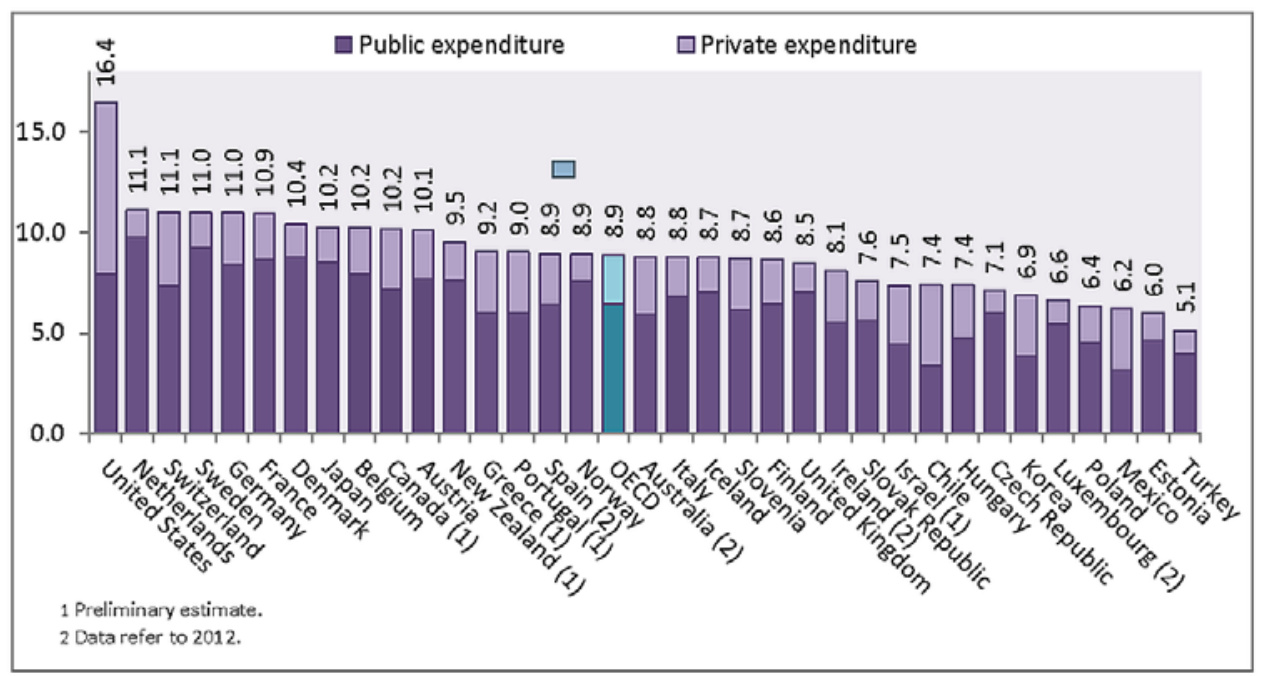

Figure 1. Health spending (excluding investment) as a share of GDP, OECD countries 2013 (OECD Health statistics 2015)
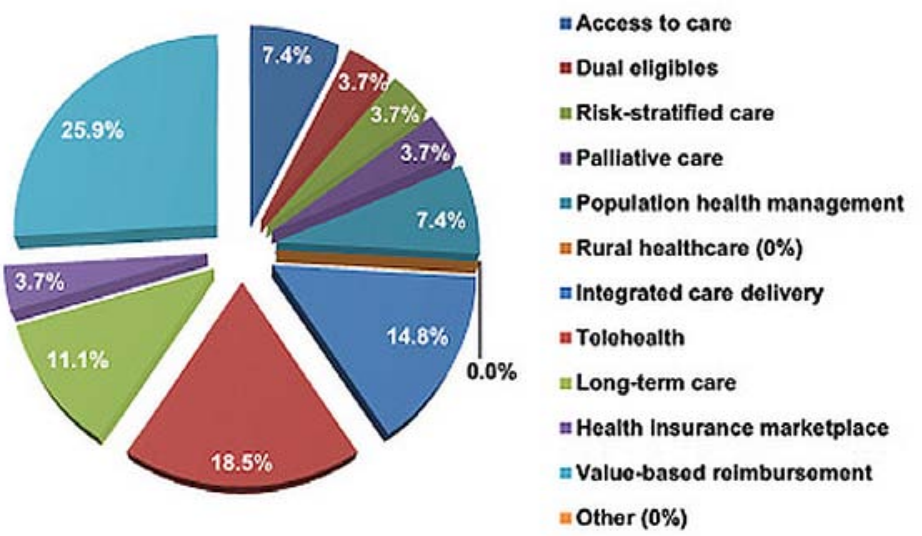

Figure 2. Top Healthcare development areas for 2015 (HINtelligence report, 2015)

The provision of high-quality, affordable, healthcare services, is an increasingly difficult challenge and healthcare systems are usually designed to meet the healthcare needs of target populations. Achievements in health worldwide in the twentieth century and particularly during the past few decades are impressive. The gains in health and quality of life are mainly the result of achievement and evolution of medical care industry, producing and delivering in broader parts of the population a complex of services that center about caregivers, private and group practices, hospitals and public health (Theodoropoulos, n.d.). To these health gains, the rapid advancements in information and communications technologies (ICT) and the increasing number of smart things (portable devices and sensors) have contributed by shifting an old-fashioned healthcare system to a model better suited for a population of the $21^{\text {st }}$ century. A healthcare practice supported by electronic processes and communication is usually referred to as e-health. E-health nowadays is closely related to the Internet (Niewolny, 2013). The rapid development of technology and the Internet leads to growing applications of novel technological solutions at the global level. E-health systems can deliver highquality information in real time and in a manner that is easily accessible everywhere, while at the same time they have the power if not to reduce, at least to manage this complexity. For example, caregivers can access patients' medical records more easily, get quick access to test results from the laboratory, and deliver prescriptions directly to pharmacists (Mukhopadhyay and Postolache, 2013). In summary, some of the most promising use cases of connected e-health include preventive health, proactive monitoring, follow-up care and chronic care disease management.

To enable healthcare professionals to track patients more continuously, and allow patients to avoid visiting facilities for everyday routine testing, nowadays there exist numerous solutions based on wearable mobile medical devices which allow patients to capture certain medical data. These, self-monitoring 
healthcare devices exist in many forms. As Do It Yourself (DIY) healthcare became the top healthcare trend of 2015 (Comstock, 2015) this paper is focused on the creation of such system which will include everything user would expect from a commercial system. In other words, by using inexpensive hardware and open source software, it is possible to create a DIY system in such a way that own solution meets user's specific needs. The proposed solution can be used to monitor human vital parameters as well as some of the environmental parameters affecting health. Therefore, providing techniques to end-users and the possibility to shape products according to their needs is beneficial for both users and product developers.

The rest of the paper is structured as follows. Section 2 presents a state of the art and emphasizes the importance of novel technologies uses in healthcare. A proposition of the DIY system for monitoring health parameters as well as environmental parameters influencing health is given in Section 3. Section 4 considers security and privacy aspects of IoT healthcare systems. Section 5, concludes the paper.

\section{STATE OF THE ART}

The social, economic, cultural and physical environment, in which people live, has a significant effect on their health and wellbeing. Therefore, the human health is directly or indirectly affected by many factors like society, family, friends, living and working conditions, education, culture, environment, economy, etc. Nevertheless, the person's individual characteristics and behaviors play the important role in determining an individual's health. Monitoring of patient's vital parameters is performed to help the evaluation of person general physical health, give hints to possible diseases, and show progress toward recovery. The rapid technology development is followed by novel devices, brought out to patients in many forms, which enable tracking health information that may be of vital importance for some patients. Nowadays there are a variety of devices in the market which are able to remotely monitor patient's vital parameters like: temperature, electrocardiogram (ECG), blood pressure, blood sugar, pulse rate or oxygen saturation level. The objective of using sensors in or around the body is to collect signals corresponding either to physical activities or to physiological conditions of the user. A list of common sensor types employed in body area network devices includes (Cayirci, 2013):

- Inertial motion sensors (employed to estimate and monitor body posture and miscellaneous human motion patterns);

- Bioelectrical sensors (e.g. ECG sensors, EMG (electromyography) sensors);

- Electrochemical sensors (e. g. blood glucose sensor, carbon dioxide concentration levels sensor);

- Optical sensors (e.g. measurement of oxygen saturation); and

- Temperature sensors.

The new model of healthcare nowadays is mostly applied to three highly prevalent chronic disorders that are predicted to become the top three causes of mortality in the year 2020 (cardiovascular, neurologic and respiratory disorders). The patients are normally equipped with wearable light devices, capable of acquiring healthcare data, and subsequently transfer them to a local processing subsystem at the patient's home via some communication links (Bluetooth, NFC or RFID) (Figure 3).

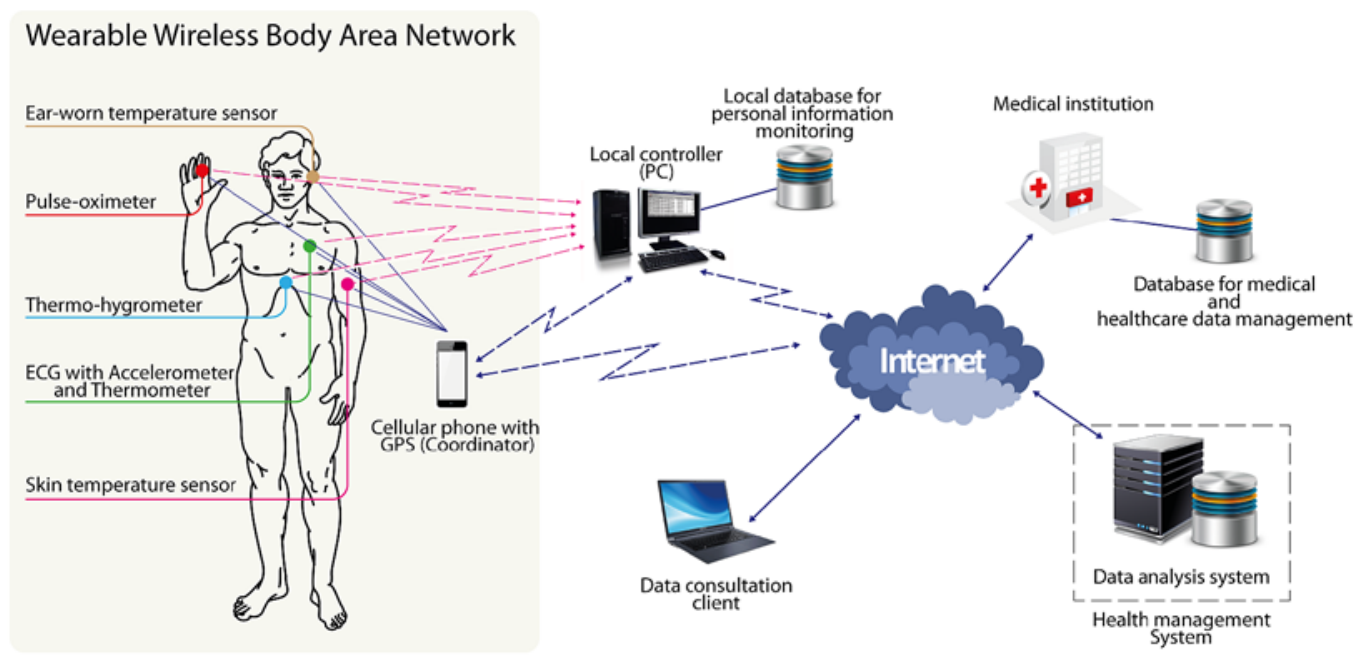

Figure 3. System concept for pervasive healthcare (Mukhopadhyay and Postolache, 2013) 
The local processing subsystem may simply transmit the data to the remote system (clinic/networked healthcare professionals) (via GSM or WiFi), or it may apply complex processing on data (e.g. artificial intelligence), before sending it. In the first case the remote subsystem stores and processes the data and creates alerts when the patient's condition becomes critical while in the other case, the local processing subsystem runs special software to analyze the acquired data. In the case of any abnormality the findings and the collected vital data are immediately transmitted to the healthcare givers for further analysis as well as alerts being produced. In summary, patient telemonitoring, signal and image processing, alert and alarm system are crucial in saving lives (Nakashima et al., 2010). Well known pervasive healthcare systems are summarized in (Nakashima et al., 2010):

- @Home system which is able remotely to monitor patient's vital parameters;

- HEARTFAID which is able to improve early diagnosis and to make more effective the medicalclinical management of heart diseases within elderly population;

- ALARM-NET is an Assisted-Living and Residential Monitoring Network for pervasive, adaptive healthcare;

- CAALYX (Complete Ambient Assisted Living Experiment) which increases older people's autonomy and self-confidence by developing a wearable light device for measuring particular vital signs of the elderly, detecting falls, and communicating automatically in real time with his/her care provider in case of an emergency, wherever the elderly person happens to be, either at home or outdoors;

- TeleCARE integrates the Internet with agent and mobile-agent technologies to develop a configurable common infrastructure so that the elderly people and those responsible for providing care and assistance can get the best out of the technologies developed;

- CHRONIC (An Information Capture and Processing Environment for Chronic Patients in the Information Society) is a new European model for the care of targeted chronic patients based on an integrated IT environment;

- MyHeart is an intelligent system for the prevention and monitoring of cardiovascular diseases using smart electronic and textile systems and appropriate services that enable the patients to take control of their own health condition;

- The OLDES is an innovative low-cost technological platform, which provides a wider range of services to elderly people; and

- The SAPHIRE which enables the patient monitoring by using agent technology complemented with intelligent decision support systems based on clinical practice guidelines. The observations received from wireless medical sensors together with the patient medical history is used in the reasoning process.

Similarly, the work described in (Mukhopadhyay and Leung, 2010) demonstrates smart, intelligent, wireless sensor devices that can be used to perceive utilization of appliances necessary for daily living and in this way determine the life style of elderly person living alone. The system developed in this work uses a limited number of sensors and can be installed/maintained in residential environments easily.

Today's personal health monitoring systems and similar medical devices must utilize an assortment of design techniques to protect the underlying design as well as protect the delicate information stored within or transmitted to/from the device (Wilkowska and Ziefle, 2012). Many personal health monitoring devices must also be portable, hence they need to be small, lightweight, and low-power. For instance, ultra-cheap-yetserviceable, small and powerful computer board - Raspberry Pi (RPi) has built in support for a large number of input and output peripherals and network communication, and it is the perfect platform for interfacing with many different devices and utilizing as a part of an extensive variety of uses (Raspberry Pi, n.d.). Its Sensor Shield V2.0 (SSV2.0) performs biometric and medical applications where body parameters are observed by using 10 different sensors: pulse, oxygen in the blood (SpO2), airflow (breathing), body temperature, electrocardiogram (ECG), glucometer, galvanic skin response (GSR - sweating), blood pressure (sphygmomanometer), patient position (accelerometer) and muscle/electromyography sensor (EMG). Such system enables real time monitoring the state of a patient or getting sensitive data in order to be subsequently analyzed for medical diagnosis. The biometric information gathered can be wirelessly sent using any of the six connectivity options available: Wi-Fi, 3G, GPRS, Bluetooth, 802.15.4 and ZigBee depending on the application (Sensor Shield V2.0, 2013). Using an RPi's support for DSI (Display Serial Interface) and for CSI (Camera Serial Interface) solution easily can be expanded to display and camera, respectively. 
The current state of sensor, processing and communication technologies, joined with comparatively low priced hardware make it possible not just to measure vital parameters but also to equip a living environment with systems of communicating sensor devices (Figure 4) (Kröse et al., 2011).

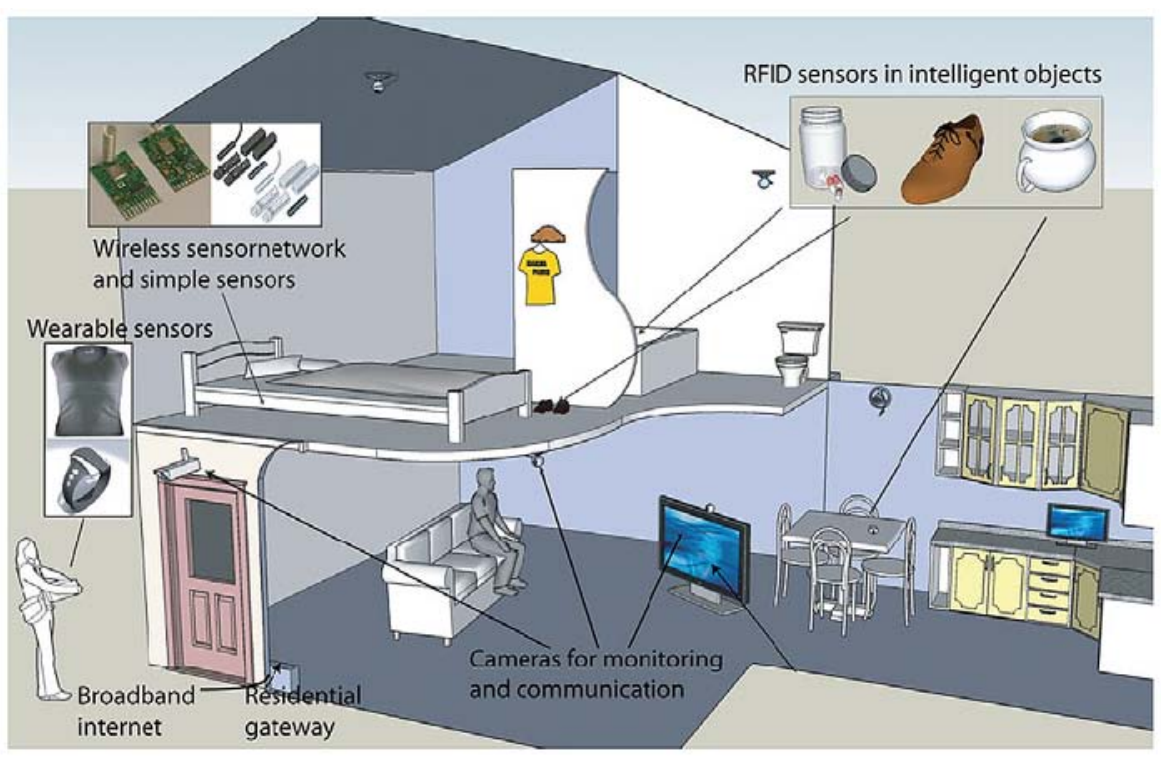

Figure 4. Sensors utilization in health monitoring and communication (Kröse et al., 2011)

Environmental factors affect large groups that share a common living or working spaces and may influence psychological conditions. In general, the factors in the physical environment that are important to health include (Determinants of Health, n.d.):

- Natural surroundings, such as plants, weather, or climate change;

- Built environment, such as buildings (worksites, schools, homes, street connectivity...), physical barriers (especially for people with disabilities) or transportation;

- Exposure to toxic substances and other physical hazards.

Airborne exposure to formaldehyde, carbon dioxide, ozone, nitrogen dioxide, methane, pollen, temperature, relative humidity, radiation, total volatile organic compounds and many other physical environmental factors have a huge influence on health. Further, extreme hot or cold may trigger some disorders, like stroke, house dust or pollen may cause allergic effects, noise may cause sleep disorders and so on.

Observing of environmental parameters for healthcare has not been attempted extensively, but it would not be hard to perform utilizing the conventional sensors. Appliance of existing sensor technologies creates many possibilities for home monitoring of health parameters. Uses of advanced sensor technologies have been attempted, but even simpler and primitive sensors can be effectively applied in daily activity monitoring. For instance, the temperature and humidity of each room can be monitored easily. Sound and vibration can be observed in different frequency ranges. Illumination in each room can also be supervised. Smoke detectors are installed in buildings and houses for detecting fire while carbon dioxide can be monitored by a simple infra-red-absorption type carbon dioxide sensor (Öberg et al., 2004). Therefore, the above presented approaches can be also used in measuring these environmental parameters, using adequate type of sensors, as it is shown in (Cayirci, 2013). The chosen sensors must be non-invasive, easy to set up and use, with effective daily activities, noise and light nuisance, waterproof, stable and reliable performance on wireless or wired minimal transmission, and to have the minimal impact on the user's normal life, which meet the medical sensor requirements.

Compared to the use of a large number of wireless sensors, the strategy of deploying a few but highly accurate numbers of inexpensive, smart and intelligent sensors has significant advantages in case of home monitoring. The fundamental point is to position sensors near to the source of a potential problem phenomenon, where gained data is likely to have the greatest benefit or impact. To justify their existence and purpose in the networked system, a sensor must fulfill a quantifiable and verifiable set of requirements that will demonstrate its benefit and purpose to the system (Mukhopadhyay and Leung, 2010). Up to date, a lot of observing instruments for home care and telecare have been proposed and created and some of them are 
turning out to be commercially available. Some instruments do not require sensor connection to the body and are completely programmed so that health parameters can be monitored without performing any operation. Using such instruments, and by associating to the telecommunication network system, physical condition of patients of chronic diseases as well as the daily life of elderly people living alone can be monitored from distant places, and collected data can be analyzed at a central station. Consequently, technologies of monitoring health parameters at home are now becoming available, and long-term monitoring data will be collected which are hardly acquired by traditional examinations in hospitals (Öberg et al., 2004). Such approach creates an opportunity for smart devices to deliver more valuable data, decreasing the necessity for direct patient-healthcare professional interaction. With faster, better insights, healthcare professionals can improve patient care, chronic disease management, hospital administration and supply chain efficiencies, and provide medical services to more people at decreased expenses.

\section{The future of healthcare systems}

The appearance of the Internet of Things (Io'T), defined as a highly dynamic and radically distributed networked system, composed of a very large number of smart objects, offer promising solutions for healthcare, creating a more revolutionary archetype for healthcare industry developed on a privacy/security model (Pang, 2013). In other words, including and applying IoT concepts in healthcare system, implies the possibility of its application for saving lives virtually unlimited (Venkatramanan and Rathina, 2014). IoT powered e-health solutions should provide a great wealth of information to the patients and their doctors that can be used to make actionable decisions, regardless of their location (Lake et al., 2014). The IoT has already brought in significant changes in many areas of healthcare (Brasseal, 2015):

- Patient monitoring and diagnostics;

- Information and data transfer, storage, and collaboration;

- Intelligent healthcare devices and tools (smart wheelchair, RFID, sensors); and

- Connected emergency units, response vehicles, and hospitals.

McKinsey Global Institute in its report presents predictions and economic feasibility of IoT powered healthcare, which states that by 2025 the largest percentage of the IoT incomes will go to healthcare (Figure 5).

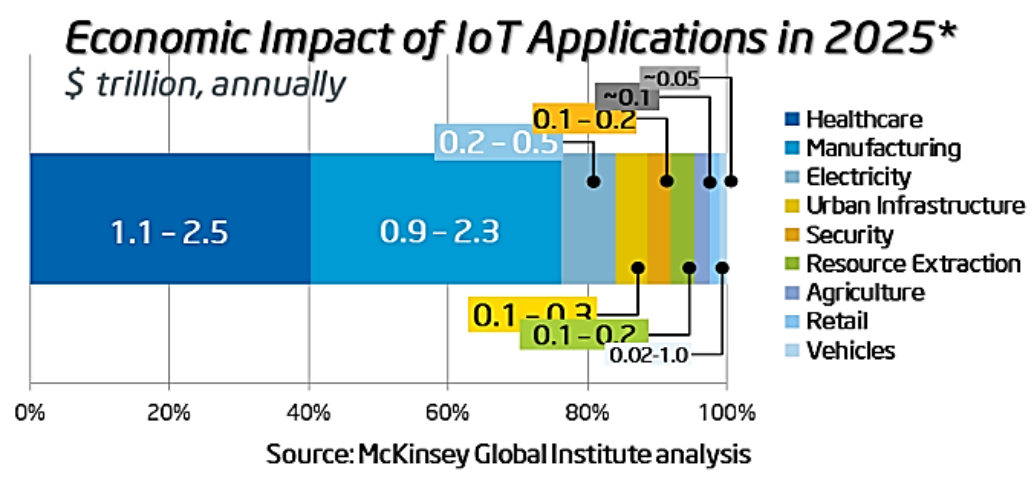

Figure 5. Economic Impact of IoT Applications in 2025 provided by the McKinsey Global Institute

According to a report by the Atlantic Council there are four categories of medical devices that meet the IoT criteria: (Glaser, 2014):

- Consumer-based (e.g. fitness tracking devices);

- Wearable, external devices (e.g. insulin pumps);

- Internally embedded devices (e.g. pacemakers and implantable cardio defibrillator devices, miniaturized (within the body) sensors); and

- Stationary devices (e.g. home-monitoring devices, IV pumps and fetal monitors).

In the recent years, there is the emergence of connected medical devices such as smart heart rate monitors, blood pressure cuffs, glucometers, asthma inhalers, thermometers and pill bottles. Internetconnected medical devices and applications are commonly referred to as the Internet of Medical Things (IoMT) (Stein, 2015). It can be stated that the IoMT as well as IoT, is a disruptive innovation, which bridges interoperability challenges to radically change the way in which healthcare will be delivered, driving better outcomes, increasing efficiency and making more cost-effective, higher quality and better-personalized care 
(IoT Healthcare, n.d.). The new generation of "medical" or "clinical wearables" is going to be equipped with more sophisticated sensing, capture and analytical functionalities, thus making the clinical usefulness of those devices more actionable. Frost \& Sullivan's recent study on consumer behavior to digital health shows approximately $24 \%$ of consumers currently use mobile apps to track health and wellness, $16 \%$ use wearable sensors and $29 \%$ use electronic personal health records. This tendency is expected to continue as $47 \%$ of consumers would consider using wearables in the near future (Das, 2015). Examples of IoMT include remote patient monitoring of people with chronic or long-term conditions; tracking patient medication orders and the location of patients admitted to hospitals; and patients' wearable mobile health devices, which can send information to caregivers. Infusion pumps that connect to analytics dashboards and hospital beds rigged with sensors that measure patients' vital signs are medical devices that can be converted to or deployed as IoMT technology. The practice of using IoMT devices to remotely monitor patients in their homes is otherwise called telemedicine. Hospitals use IoT to keep tabs on the location of medical devices, personnel and patients. This sort of treatment spares patients from traveling to a hospital or physician's office whenever they have a medical inquiry or change in their condition.

Furthermore, change in the healthcare system in total, has also been contributed by the rapid development of nanotechnologies. IoT with nano-machines have attracted much attention as one of the new research areas, giving to the healthcare system a new, global domain - Internet of Nano Things (IoNT) and nanomedicine. The application of IoNT in nanomedicine presents a very significant improvement in nanomedicine - enhancing human health in novel ways, particularly in preventive health, proactive monitoring, follow-up care and chronic care disease management. In other words, through the design, characterization, production and application of nano sized, intelligent materials and their application within medicine, there is a possibility to revolutionize the healthcare (Omanović-Mikličanin et al., 2015).

It is evident that patients and caregivers, both stand to benefit from IoT/IoMT/IoNT carving out a bigger presence in healthcare. Adequate ways and means to ensure the appropriate healthcare delivery are based on parameters monitoring and direct providing of the medical assistance. In order to track and record, in real time manner, personal data as well as surrounding parameters influencing health, it is necessary to use adequate devices or tools which are readily available to the general public. Such devices are usually wearable and the tools are digitally available through mobile device applications while the scale of nanodevices enables reaching hard to access areas and access to vital information at a whole new level (molecular information). Nanomachines deployed inside the human body, as well as various medical devices and sensors, can be remotely controlled from the macroscale and over the Internet by an external user (a healthcare provider). In summary, the concept of the IoT/IoMT/IoNT entails the use of electronic devices that capture or monitor data and are connected to a private or public cloud, enabling them to automatically trigger certain events. These concepts are rapidly changing the healthcare scenario by focusing on the way people, devices and applications are connected and interact with each other. Ultimately, everyone in the care team will be connected (Figure 6).

IoT functionality derives from the interactions between three architectural layers: information perception layer (sensors tasked with data collection, e.g. wearable physiological parameter detection sensors, home environment and behavior detection sensor), network (transformation) layer (all kinds of wire or wireless computer network) and the application layer (service center, medical personnel mobile terminal and patient mobile terminal) (Li, 2011; Zhao, 2013).

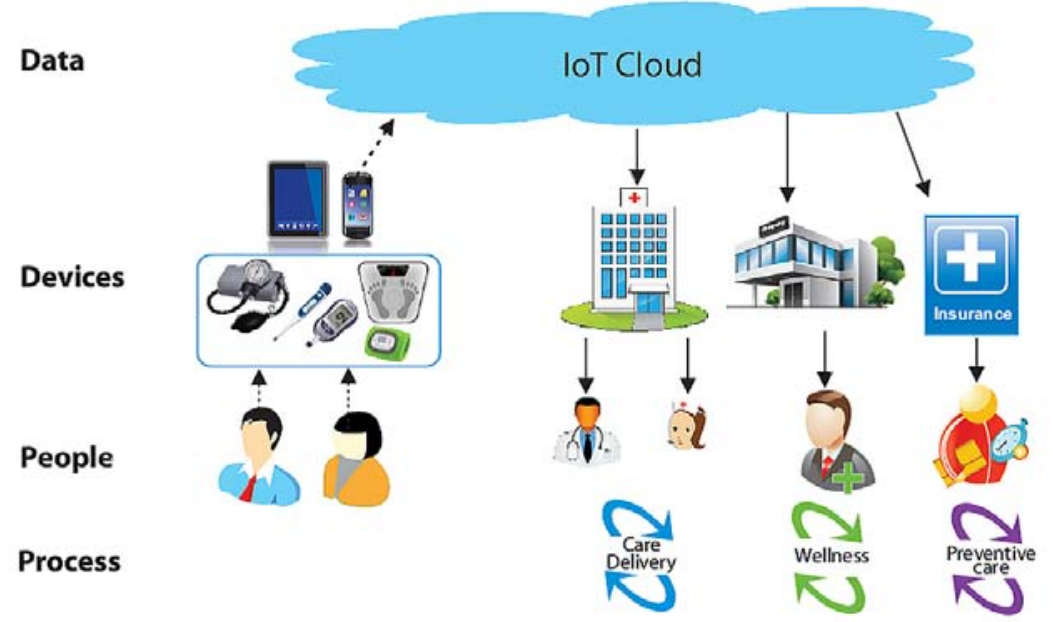

Figure 6. The confluence brought about by the IoT (IoT Healthcare, n.d.) 
The performed survey confirms that the convergence of IoT, health data, biomedicine, wireless, and mobile have transformed medicine from an art to a data driven science, providing the right care, in the right place, at the right time and at affordable cost (Deloitte, n.d.). Therefore, the IoT solutions for healthcare are key factors to re-imagining patient care. Some of their impacts include (Kramer, 2015): better patient care, greater operational efficiency and reduced cost and enhanced asset monitoring and tracking. The relationship between the healthcare industry and the IoT is a promising one, but it's not without obstacles. Kramer (2015) defines a few key challenges facing this union: mobile hesitation, consistency issues and security questions, what will be discussed in the last chapter.

\section{DIY HEALTHCARE SYSTEM DESIGN}

Today, the greatest challenge in healthcare is how to enable doctors to track people more continuously, and allow patients to avoid visiting facilities for routing testing. A solution can be found in mobile medical devices which allow healthcare providers to transfer some basic monitoring tasks to patients as selfmonitoring devices. The self-monitoring devices are created for the purpose of allowing personal data to be instantly available to the individual to be analyzed, and as such these devices are an important advancement in the field of personal health management (Maksimović et al., 2015). Typically, IoT powered e-health solution includes the following functions (Pang, 2013):

- Tracking and monitoring (e.g. patient monitoring, chronic disease self-care, elderly persons monitoring or wellness and preventive care);

- Remote service;

- Information management; and

- Cross-organization integration.

The greatest benefit of self-monitoring devices is the elimination of the necessity for third party hospitals to run tests, which are both expensive and lengthy. The additional advantage is connection with healthcare expert systems which are very important for giving correct information for diagnosis, reducing medical errors and providing prompt medical services (Maksimović et al., 2015; Omanović-Mikličanin et al., 2015).

DIY healthcare, including mobile applications and consumer medical devices, is the one of the top healthcare trends today. Many healthcare providers prescribe mobile applications for patient management, particularly for things like healthy nutrition, weight loss and exercise. In the near future there are expectations that the emphasis is on flexibility, convenience and technologies that deliver personalized experiences that meet user's specific needs (Comstock, 2015). According to this, the focus of this paper is on the creation of custom system which will include everything user would expect from a commercial system. In other words, by using inexpensive hardware and open source software, it is possible to create a DIY system in such a way that own solution meets user's needs and emphasize well-being. It is important to note that when developing or using health, wellness, or environmental devices, it is important to be aware of the regulations that pertain to that device and ensure that the device is compliant. In other words, healthcare devices require a degree of regulation to ensure that they are electrically, chemically, biologically, and physically safe for the end user. The topics of standards and regulations applied to sensor-based health, wellness, and environmental domains are presented in (McGrath and Ni Scanaill, 2013).

\section{Building a prototype}

Currently, self-monitoring healthcare devices exist in many forms. A different technologies and architectures of IoT for healthcare can be found in various papers (Mukhopadhyay and Lay-Ekuakille, 2010; Nakashima et al., 2010; Salvi et al., 2010; Nugent et al., 2013; Pang, 2013; Lake et al., 2014; Mukherjee et al., 2014; Adibi, 2015), but next building elements are common for all of them (Niewolny, 2013):

- Sensors that collect data (e.g. medical sensors attached to the patient to measure vital parameters, and the environmental sensors which monitor the surroundings of the patient);

- Microcontrollers that process, analyze and wirelessly communicate the data;

- Microprocessors that enable rich graphical user interfaces; and

- Healthcare-specific gateways through which sensor data is further analyzed and sent to the cloud.

Relying on the publications (Maksimović et al., 2014; Vujović and Maksimović, 2014) where the discussion of the use of small, cheap and flexible computer hardware is performed, the key element for building a custom IoT healthcare solution is a Raspberry Pi (RPi), fully customizable and programmable small computer board. The RPi has built in support for 26-pin GPIO (General Purpose Input and Output) port which can be used as 
digital input/output signals and can be programmed directly on RPi through high level programming languages like $\mathrm{C}++$, Python and Java. Some of the GPIO's pins can be used as interfaces for embedded protocols for controlling a set of electronic circuit (sensors, analog to digital converters (ADC), relay, status button, etc.). Therefore, the GPIO port is the main way of connecting RPi with other electronic boards. Via GPIO port it is possible to communicate with other computing devices using a variety of different protocols, including Serial Peripheral Interface (SPI), Inter-Integrated Circuit $\left(\mathrm{I}^{2} \mathrm{C}\right)$ and Universal Asynchronous Receiver/Transmitter (UART). The primary disadvantage of RPi is the absence of the ADC, which can be removed using a cheap external ADC such as PCF8591 8-bit I2C or MCP3008 10-bit SPI devices.

Two examples of proposed the DIY system are considered in this paper, a measurement of body and environmental parameters. The main aim of presented system is the development of collection of modules which can facilitate the diagnosis for the doctors through patients telemonitoring.

The main application domain of a network of sensors attached to the human body is continuous health monitoring and logging vital patients' parameters. The proposed solution enables observing body temperature, blood pressure and heart rate (Figure 7). Therefore, the following sensors are used in the custom solution:

- Temperature sensor (TTC05) - detection of body temperature and its changes are vital for the people's health;

- Heart rate sensor (Pulse sensor module) - detection of body heart rate or pulse is important for people's health, weight lost and exercise; and

- Blood pressure sensor (US9111) - used to detect blood pressure and prevent health hazards like heart attack and stroke.

In the other case, temperature, humidity, air quality and light intensity, as environmental parameters, are observed by using following sensors:

- Temperature sensor (TTC05) - detection of environmental temperature and its changes are vital for the people's health;

- Humidity sensor (RH31) - detection of relative humidity provides an environmental analysis of increased humidity;

- Air quality sensor (MQ2) - detects the presence of substances such as benzene, alcohol, smoke, CO and $\mathrm{CO}_{2}$. Some of these substances are highly carcinogenic, and their presence is not desired in the objects people reside or work; and

- Light intensity sensor (LM393) - used to detect the intensity of light.

Experimental sensor web node scheme with four sensors is shown below (Figure 8).

The aim of the proposed system is the usage of a limited number of sensors that can be easily installed/maintained in environments in which people reside or work. The additional system advantage lies in easily installation in an existing home/work environment with no major modifications or damage.

Regardless of the considered case, these networked systems continuously monitor patients' physiological and physical conditions or environmental parameters affecting health, and transmit sensed data in real time via either wired or wireless technology (WiFi/GPS/GSM) to a centralized location where the data can be monitored and processed by trained medical personnel (Figure 9).

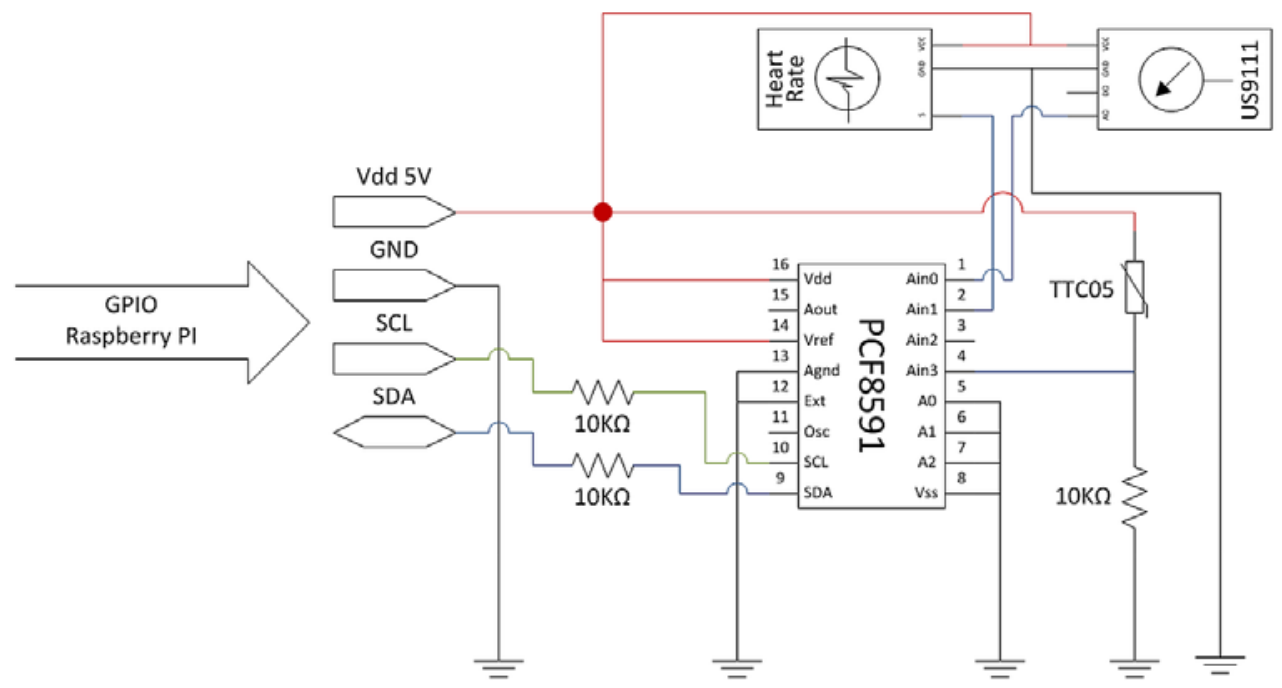

Figure 7. Electronic circuit scheme of Raspberry Pi as a sensing unit for measuring vital parameters 


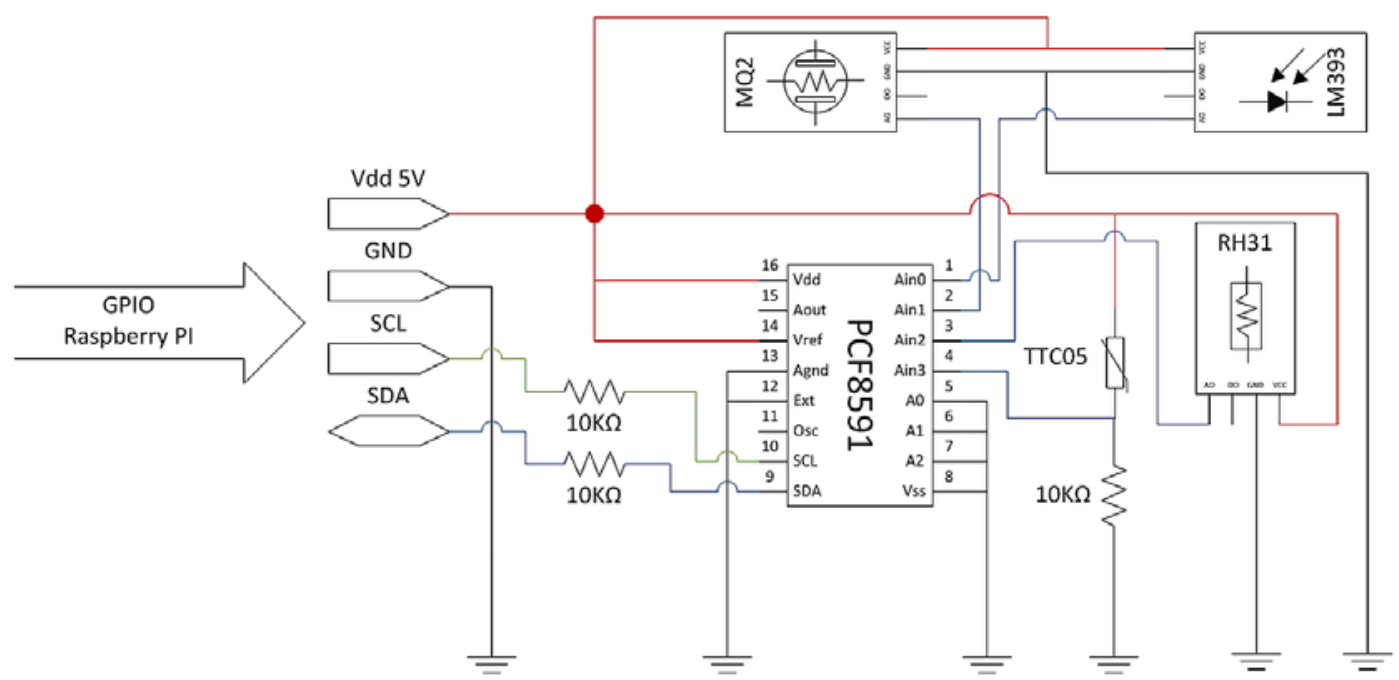

Figure 8. Electronic circuit scheme of Raspberry Pi as a sensing unit for measuring environmental parameters

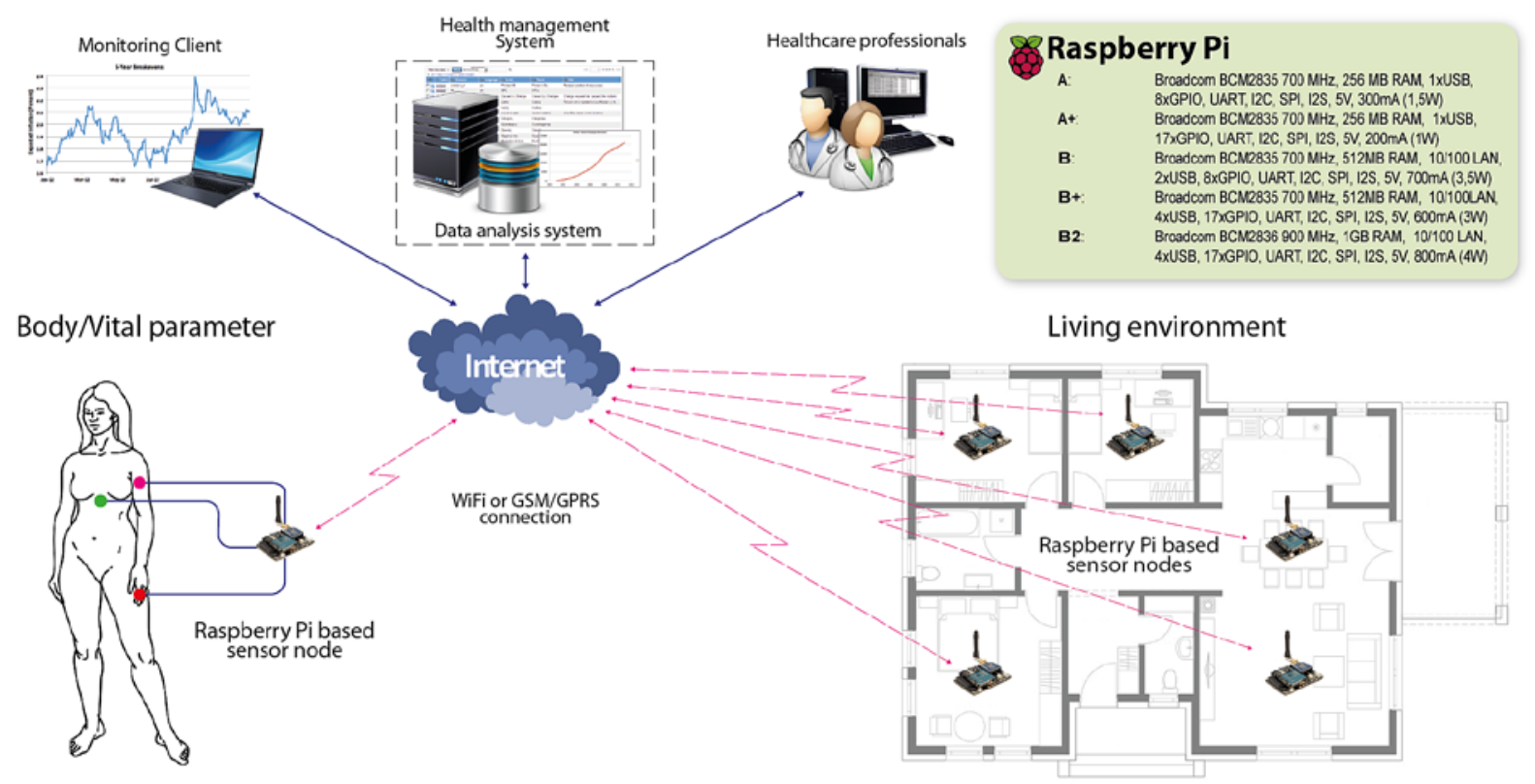

Figure 9. Monitoring vital parameters and environmental parameters affecting health using proposed DIY system

For delivering measurement information to the user or healthcare providers and comply with IoT ideology, a DIY solution based on RPi must implement one of the HTTP WEB Server (in our proposed solution, Apache Tomcat) which can provide a lightweight REST services (Fielding, 2000). In the case of applying a REST service to IoT solution, every action represents a resource and has a unique URI address via which is accessed to the sensor data. For instance, for the body temperature readings from sensor, a URI address is provided:

\section{(server adress)/RPIHealthCare/sw/temp}

which generates a JSON (JavaScript Object Notation) response:

\{"temp": "36.5"\}

Since data from sensor node are obtained in JSON format, a different client application for visualization and processing can be created based on custom user's need. The cost of this proposed DIY solution depends on quality of sensors (different manufacturer gives a different quality of hardware) and resolution of ADCs. 
Sensed data can be relayed to the server or a base station in close proximity. In such way, the doctors and caregivers monitor the patient in real time through the data received through the server. The medical history of each patient, including medications and medical reports are stored in a central database in the cloud or visualized in real time for easy access and processing for logistics and prognosis of future complications. The data can be processed in two ways, on-board processing (allows immediate detection anomalies and care could be taken before the patient reaches healthcare institutes) and on server processing (uses the real time metadata received from the sensors to process them with respect to the data stored in the cloud itself). On server processing requires better resources in the form of memory, throughput and processing time and hence is more suitable (Mukherjee et al., 2014).

Having in mind that IoT is an issue of scale and that scale affects everything, massive volumes of disparate data, typically dimensioned by space and time, new devices and protocols, burst traffic, a heavier reliance on the cloud and increased IPv6 deployment are five key impacts necessary to be considered in IoT vision. In IoT there's a tension between effort, efficiency and time-to-market, and generally there's a trade-off between them: it is possible to have any two but not all three. Connected devices, sensors, wearables, and other non-human stuff produce massive quantities of data and therefore, sensor data bring numerous challenges with it in the context of data aggregation, storage and processing. When viewed on a device-by-device basis, the bulk of data produced are not things of concern. Nevertheless, when submitted in aggregate, this data puts a strong threat to specific networks and the systems that manage them. The solution is to consider a monitoring platform based on a distributed computing model. In other words, keeping performance data distributed across the network, it is easier to manage the challenge of massive data generated by the IoT (Maksimović et al., 2014). Getting crystallized information which can be applied to make fast and accurate predictions and decisions is usually done through the computational process using artificial intelligence, machine learning, statistical analysis and database systems.

\section{SECURITY AND PRIVACY CONCERNS OF IOT POWERED HEALTHCARE SYSTEMS}

The realization of the IoT generally requires dramatic changes in systems, architectures and communications which should be flexible, adaptive, secure, and pervasive without being intrusive (Yan et al., 2008). The experts anticipate that the technologies of the IoT will bring forward critical developments in the areas of ethics, data protection, technical architecture, standards, identification of networked objects and governance (Meier-Hahn, 2013). As more intelligent devices are linked to the Internet, the potential privacy implications and general false sense of security associated with weak key management and data compromise become critical. The security issues (protection of data and privacy) may arise both during data collection and during data transmission and sharing and therefore represents a critical component for enabling the widespread adoption of IoT technologies and applications.

The rapid development of IoT healthcare contains the risk of security and privacy. Thus, to develop and integrate effective ubiquitous sensing for healthcare, security and privacy as important design goals should be taken into account. The official breach reports from 2009 to mid-April 2013, presents the security risks for healthcare IT professionals (Figure 10) (HIPAA, 2013).

To reduce and solve the risk of security and privacy in healthcare applications, a number of literature articles were published (Appari and Johnson, 2008; Löhr et al., 2010; Shih and Zhang, 2010; Tsai, 2010; Wilkowska and Ziefle, 2012; Agrawal, 2014; Kim, 2014; Lake et al., 2014, Waegemann, n.d).

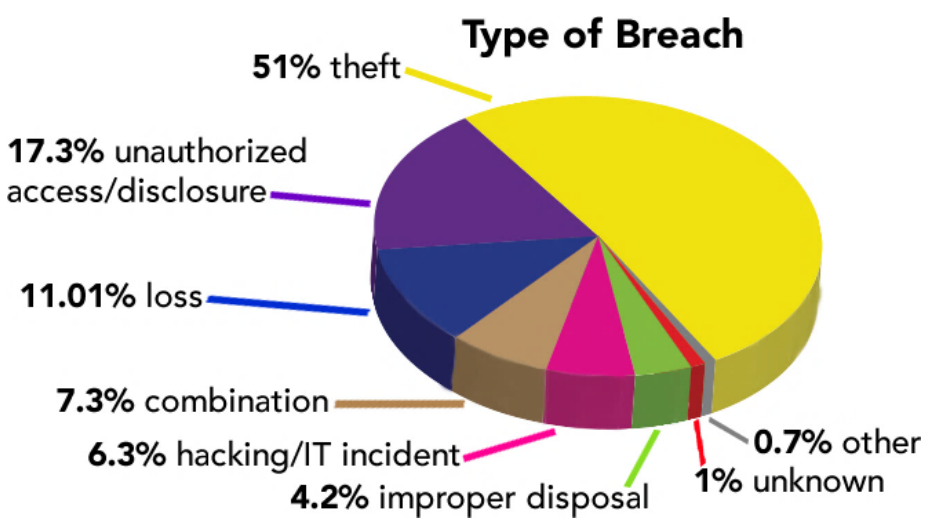

Figure 10. Security risks for healthcare IT professionals (HIPAA, 2013) 
The requirements of IoT communication framework in e-health applications can be summarized in (Bui and Zorzi, 2011):

- Interoperability - is needed to enable different things to cooperate in order to provide the desired service.

- Bounded latency and reliability - are needed to be granted when dealing with emergency situations in order for the intervention to be effective.

- Authentication, privacy, and integrity - are mandatory when sensitive data are exchanged across the network (Figure 11).

\begin{tabular}{l|ll|}
\hline Authentication & Data Integrity & System Security \\
- Identification & - Encryption & - Communication \\
- Signature & - Data Integrity & - Processing \\
- Non-repudiation & Process & - Storage \\
& - Permanence & - Permanence
\end{tabular}

Internet Security
- Personal Health
Records
- Secure Internet
Services

Figure 11. Healthcare specific security standards (Waegemann, n.d.)

User authentication is very important in healthcare information systems as it is the first step in the entire health information access procedure. Some of authentication mechanisms are needed to verify the user's identity like: password, PIN, fingerprint, signature, voice pattern, smart card, token, etc. In this way we can be sure that the information is sent by the trusted sender. The next important factor is the data access control, which prevents unauthorized accesses to the patient's data. With patient data integrity, the transmission of medical reports from the patient to the medical staff cannot be modified or changed by any outside or unauthorized source. Also, the patient's records cannot be interpreted by anyone other than the authorized medical personnel, therefore confidentiality ensures that a patient's medical files are protected from passive attacks such as eavesdropping or traffic analysis (Divi et al., 2010). The data being generated, gathered, and shared through networked devices must be protected with strong, usable authentication methods. As some of collected data may be private and patients don't want to share them with others, even clinic physicians, the disclosure of such private data could lead to unwanted privacy threats to the patients (Shih and Zhang, 2010). Thus, portable devices to record, store, and transmit patient data to a central server, need secure ways to transmit recorded data at high speed to ensure that personal records can't be compromised at any point. Inevitably, adequate regulative and standards, like the ISO/IEC 27000-series which comprises information security standards, must be satisfied.

In summary, security should be built into the whole healthcare ecosystem, from the device, to the network, to the data center. A strong security strategy includes authentication technologies and processes to verify patient and provider identities to ensure that devices can only be used by authorized users. The communications channels between the devices within the IoT must also be secure to ensure the integrity of the information passing through them (Ting, 2015).

As e-health systems store and process very sensitive data, they should have a proper security and privacy framework and mechanisms. Figure 12 shows the link between many important healthcare research problems and information security (Appari and Johnson, 2008).

Protecting privacy must not be limited to technical solutions (encryption, ID management, privacyenhancing technologies), but encompass regulatory (consumer consent, collection limitation, openness, accountability), market-based (self-regulation, privacy certification, consumer education) and socio-ethical considerations (consumer rights, public awareness, disclosure, consumer advocacy) (Weber and Weber, 2010; Hasić and Vujović, 2014).

However, information security, privacy and data protection should systematically be addressed at the design stage. 


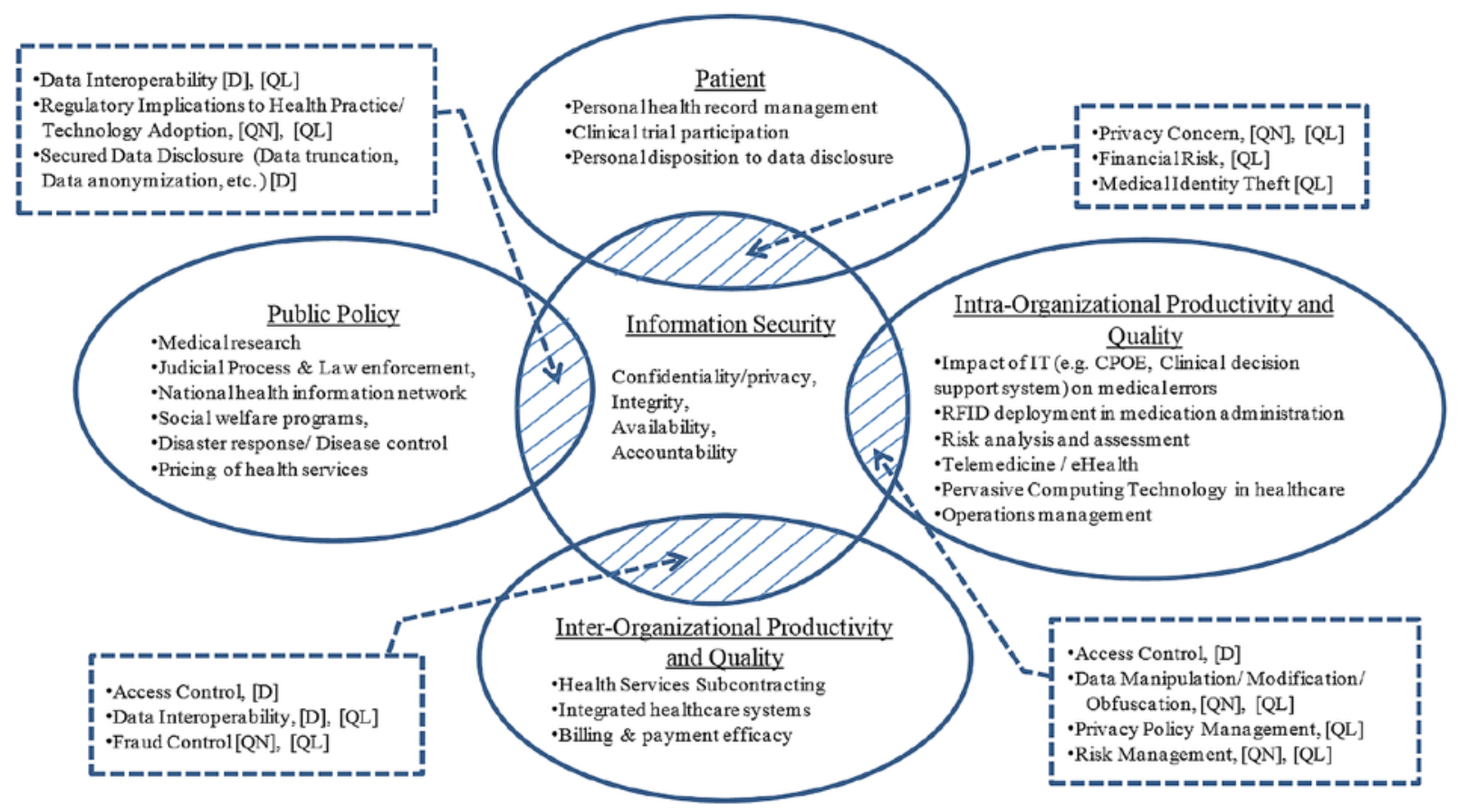

Figure 12. Research domains in the healthcare information security (Appari and Johnson, 2008)

\section{CONCLUSION}

The human health today presents the primary focus of an increasing number of case studies and projects which goal is healthcare improvements and achieving the foundations for a global health system. With the appearance of the IoT concept, elements, such as sensors and sensor networks, are becoming available and applicable in all fields of human activity, thus providing conditions for the creation of expert systems that can operate anytime and anyplace. Nowhere does the IoT offer greater promise than in the field of healthcare. By connecting information, people, devices, processes and context, IoT powered e-health creates a lot of opportunities to improve health by increasing the availability and quality of care followed with radically reduced costs. Integrating IoT features into medical devices creates a new concept, known as IoMT, which greatly improves the quality and effectiveness of service, bringing especially high value for the elderly people, patients with chronic conditions, and those requiring constant supervision. Furthermore, the advancements in nanotechnologies created a new networking paradigm - IoNT, which shows the biggest advances in the biomedical domain (diagnostic and treatment areas).

An indispensable application of IoT is in healthcare, where the application can be found in health monitoring, diagnostics and treatment more personalized, timely and convenient. All of this significantly transform the healthcare industry by increasing efficiency, lowering costs and put the focus back on better patient care. This contributes to an intelligent e-health scheme that can receive an unprecedented level of realtime, life-critical data. Analysis of captured data and provision of, in real time manner, a valuable set of information relevant to all of the stakeholders (patients, medical and paramedical stuff, and health insurance) regardless their current placement, drive efficiency, maintain compliance, and helps people advance research, management and care.

Some uses of healthcare IoT are mobile medical applications or wearable devices that allow patients to capture their health data. Additionally, observing environmental parameters affecting health is also very important for a person's health. Therefore, this paper presents two DIY solutions realized with the help of the RPi affordable prototyping platform. Both proposed solutions are built from a scratch according to specific patient's needs and offer a unique opportunity to tailor and customize care services for individual patients health needs. Without any human intervention, such IoT-enabled devices provide remote data from various connected medical devices and automatically transmit them via wearable devices. Accurately in realtime collected data means more efficiently work of hospitals through more reliable and efficient communications among caregivers and subsequently patient's better care. In other words, when a networked medical device is connected to a person, the health information that can be exchanged may dramatically 
improve healthcare. But the advantages of IoT applications in healthcare can be exploited effectively only if the desired level of security and privacy can be ensured. Therefore, presented examples show that applying technology based on IoT significantly can reduce a price of healthcare, but is also shown that there are elements (privacy, security and technology) for future improvement and consideration.

\section{REFERENCES}

Adibi, S., 2015. Mobile health. a technology road map. Springer International Publishing Switzerland.

Agrawal, V., 2014. Security and Privacy Issues in Wireless Sensor Networks for Healthcare. In Internet of Tbings. UserCentric IoT (pp. 223-228). Springer International Publishing.

Appari, A. and Johnson, M.E., 2010. Information security and privacy in healthcare: Current state of research. International journal of Internet and enterprise management, 6(4), pp.279-314.

Brasseal, M., 2015. Realtime technology and the healthcare internet of things. Available at: https://www.pubnub.com/blog/201506-19-realtime-technology-and-the-healthcare-internet-of-things/. (Accessed: 13 October 2015).

Bui, N. and Zorzi, M., 2011, October. Health care applications: A solution based on the internet of things. In Proceedings of the 4th International Symposium on Applied Sciences in Biomedical and Communication Technologies (p. 131). ACM.

Cayirci, E., 2013. Wireless sensor and actuator network applications and challenges. D. Filippini (ed.). Autonomous Sensor Networks: Collective Sensing Strategies for Analytical Purposes. (pp. 1-16.) Springer Series on Chemical Sensors and Biosensors. Springer-Verlag Berlin Heidelberg.

Comstock, J., 2015. PwC predicts "DIY Healthcare" will be the top trend of 2015. Available at: http://mobihealthnews.com/39747/pwc-predicts-diy-healthcare-will-be-the-top-trend-of-2015. (Accessed: 13 August 2015).

Das, R., 2015. Top 10 Healthcare Predictions For 2016. Available at: http://www.forbes.com/sites/reenitadas/2015/12/10/top-10-healthcare-predictions-for-2016/\#146f174c2f63. (Accessed: 18 February 2016).

Deloitte Centre for Health Solutions, n.d. Healthcare and Life Sciences Predictions 2020. A bold future? Available at: https://www2.deloitte.com/content/dam/Deloitte/global/Documents/Life-Sciences-Health-Care/gx-lshchealthcare-and-life-sciences-predictions-2020.pdf. (Accessed: 8 February 2016).

Determinants of Health, n.d.. Available at: http://www.healthypeople.gov/2020/about/foundation-healthmeasures/Determinants-of-Health. (Accessed: 1 February 2016).

Divi, K., Kanjee, M.R. and Liu, H., 2010, August. Secure architecture for healthcare Wireless Sensor Networks. In Information Assurance and Security (LAS), 2010 Sixth International Conference on (pp. 131-136). IEEE.

Fielding, R.T., 2000. Arcbitectural styles and the design of network-based software arcbitectures (Doctoral dissertation, University of California, Irvine).

Glaser, J., 2014. How The Internet of Things will affect health care. Available at: http://www.hhnmag.com/articles/3438-howthe-internet-of-things-will-affect-health-care. (Accessed: 4 February 2016).

Hasić, H. and Vujović, V., 2014. Civil law protection of the elements comprising the "Internet of Things" from the perspective of the legal owner of the property in question. Infoteh-Jahorina, 13, pp. 1005-1011.

HIPAA, 2013. Top 5 HIPAA Security Risks As Providers Migrate to the Cloud. Available at: http://cloudtweaks.com/2013/05/top-5-hipaa-security-risks-asproviders-migrate-to-tthe-cloud/. (Accessed: 22 May 2015).

HINtelligence report, 2015. Healthcare trends for 2015: Promise in payment reform, population bealth and partnerships. Available at: http://www.hin.com/library/registerHealthcareTrends2015.html. (Accessed: 10 February 2016).

IoT Healthcare, n.d. The Internet of Things and Healthcare Policy Principles, Intel. Available at: http://www.intel.eu/content/dam/www/public/emea/xe/en/documents/iot-healthcare-policy-principlespaper.pdf. (Accessed: 22 May 2015).

Kim, J.T., 2014. Privacy and Security Issues for Healthcare System with Embedded RFID System on Internet of Things. Advanced Science and Tecbnology Letters, 72, pp.109-112.

Kramer, S., 2015. How the Internet of Things will impact bealth care. Available at: https://powermore.dell.com/technology/how-the-internet-of-things-will-impact-health-care/. (Accessed: 19 January 2016).

Kröse, B., van Oosterhout, T. and van Kasteren, T., 2011. Activity monitoring systems in health care. In Computer analysis of buman behavior ( pp. 325-346). Springer London.

Lake, D., Milito, R., Morrow, M. and Vargheese, R., 2014. Internet of Things: Architectural framework for eHealth security. Journal of ICT Standardization, River Publisbing, 1.

Li, J., Wu, X. and Chen, H., 2011. Research on mobile digital health system based on internet of things. In Electrical Power Systems and Computers (pp. 495-502). Springer Berlin Heidelberg.

Löhr, H., Sadeghi, A.R. and Winandy, M., 2010, November. Securing the e-health cloud. In Proceedings of the 1st ACM International Health Informatics Symposium (pp. 220-229). ACM.

Maksimovic, M., Vujovic, V. and Perisic, B., 2015, June. A custom Internet of Things healthcare system. In Information Systems and Technologies (CISTI), 2015 10th Iberian Conference on (pp. 653-658). IEEE. 
Maksimović, M., Vujović, V., Davidović, N., Milošević, V. and Perišić, B., 2014. Raspberry Pi as Internet of things hardware: performances and constraints. 1st International Conference on Electrical, Electronic and Computing Engineering - IcETRAN 2014, pp. ELI1.6.1-6, ISBN 978-86-80509-70-9.

McGrath, M.J. and Scanaill, C.N., 2013. Sensor Technologies: Healthcare, Wellness and Environmental Applications. Apress.

Meier-Hahn, U., 2013. European Commission publishes report on Internet of things. Available at: http://policyreview.info/articles/news/european-commission-publishes-report-internet-things/114. (Accessed: 1 May 2015).

Mukherjee, S., Dolui, K. and Datta, S.K., 2014, February. Patient health management system using e-health monitoring architecture. In Advance Computing Conference (IACC), 2014 IEEE International (pp. 400-405). IEEE.

Mukhopadhyay, S.C. and Leung, H., 2010. Advances in wireless sensors and sensor networks. Berlin, Germany: Springer.

Mukhopadhyay, S. and Postolache, O.A., 2013. Pervasive and mobile sensing and computing for healthcare. Berlin, Germany; Heidelberg, Germany: Springer.

Mukhopadhyay, S.C. and Lay-Ekuakille, A. eds., 2010. Advances in biomedical sensing, measurements, instrumentation and systems. Springer.

Nakashima, H., Aghajan, H. and Augusto, J.C. eds., 2010. Handbook of ambient intelligence and smart environments. Springer Science \& Business Media.

Niewolny, D., 2013. How the Internet of Things is revolutionizing healthcare. Available at: http://www.freescale.com/healthcare. (Accessed: 25 May 2015).

Nugent C., Coronato, A. and Bravo, J. (2013) Ambient assisted living and active aging. In 5th International Work-Conference. IWAAL 2013 Carrillo, Costa Rica. December 2-6, 2013. Proceedings.

Öberg, P. A., Togawa, T. and Spelman, F. A., 2004. Home health care and telecare. In Sensors in medicine and bealth care: sensors applications. Wiley-VCH Verlag GmbH \& Co. KGaA.

OECD Health Statistics, 2015. Focus on health spending. Available at: http://www.oecd.org/health/health-systems/FocusHealth-Spending-2015.pdf. (Accessed: 7 February 2016).

Omanović-Mikličanin, E., Maksimović, M. and Vujović, V., 2015. The Future of Healthcare: Nanomedicine and Internet of Nano Things. Folia Medica Facultatis Medicinae Universitatis Saraeviensis, 50(1), pp. 23-28.

Pang, Z., 2013. Technologies and Architectures of the Internet-of-Things (IoT) for Health and Well-being. PhD Thesis in Electronic and Computer Systems. KTH - Royal Institute of Technology, Stockholm, Sweden.

Raspberry Pi, n.d. Available at: http://www.raspberrypi.org/. (Accessed: 14 February 2016).

Salvi, D., Mora, E.V. and Waldmeyer, M.T.A., 2010. An architecture for secure e-Health systems. Available at: http://www. tsb. upv. es/eventos/workshophealthcare/documentos/C2-2. pdf.

Sensor Shield V2.0, 2013. e-Health Sensor Platform V2.0 for Arduino and Raspberry Pi [Biometric/Medical Applications]. Available at: http://www.cooking-hacks.com/documentation/tutorials/ehealth-biometric-sensor-platformarduino-raspberry-pi-medical. (Accessed: 14 May 2015).

Shih, F. and Zhang, M., 2010, October. Towards Supporting Contextual Privacy in Body Sensor Networks for Health Monitoring Service. In W3C Workshop on Privacy and data usage control (Vol. 4, No. 05).

Stein, J., 2015. The Emergence of the "Internet of Medical Things." Huffpost Tech. Available at: http://www.huffingtonpost.com/josh-stein/the-emergence-of-the-inte_b_6801714.html. (Accessed: 11 February 2016).

Theodoropoulos, S., n.d.. On the effectiveness of Publicly or Privately produced Health care services. Available at: http://www.vsfs.cz/prilohy/konference/1_ws_3_1_theodopulos.doc. (Accessed: 7 February 2016).

Ting, D., 2015. The internet of things can revolutionize bealthcare, but security is key. Available at: http:/ /histalk2.com/2015/05/28/readers-write-the-internet-of-things-can-revolutionize-healthcare-but-security-iskey/. (Accessed: 12 August 2015).

Tsai, F.S., 2010. Security issues in e-healthcare. Journal of Medical and Biological Engineering, 30(4), pp. 209-214.

Venkatramanan, P. and Rathina, I., 2014. Healthcare Leveraging Internet of Things to revolutionize Healthcare and Wellness. IT Services Business Solutions Consulting.

Vujovic, V. and Maksimovic, M., 2014, May. Raspberry Pi as a Wireless Sensor node: Performances and constraints. In Information and Communication Technology, Electronics and Microelectronics (MIPRO), 2014 37th International Convention on (pp. 1247-1252). IEEE.

Yan, L., Zhang, Y., Yang, L.T. and Ning, H. eds., 2008. The Internet of things: from RFID to the next-generation pervasive networked systems. CRC Press.

Waegemann, C.P., Confidentiality and Security for e-Health. In CEO, Medical Records Institute (MRI). Available at: https://www. itu. int/itudoc/itu-t/workshop/e-health/s5-05. pdf.

Weber, R.H. and Weber, R., 2010. Internet of things: Legal perspectives (Vol. 49). Springer Science \& Business Media.

Wilkowska, W. and Ziefle, M., 2012. Privacy and data security in E-health: Requirements from the user's perspective. Health informatics journal, 18(3), pp.191-201.

Zhao, W., Lei-hong, L., Yue-shan, H., Xiao-ming, W., 2013. A Community Health Service Architecture Based on the Internet of Things on Health-Care. In World Congress on Medical Physics and Biomedical Engineering May 26-31, 2012, Beijing, China (pp. 1317-1320). Springer Berlin Heidelberg. 